\title{
Evolution of correlations of many-particle quantum systems in condensed states
}

\author{
V.I. Gerasimenko* \\ * Institute of Mathematics of the NAS of Ukraine, \\ 3, Tereshchenkivs'ka Str., 01601, Kyiv-4, Ukraine \\ E-mail: gerasym@imath.kiev.ua
}

\begin{abstract}
We review some new approaches to the description of the evolution of states of many-particle quantum systems by means of the correlation operators. Using the definition of marginal correlation operators within the framework of dynamics of correlations governed by the von Neumann hierarchy, we establish that a sequence of such operators is governed by the nonlinear quantum BBGKY hierarchy. The constructed nonperturbative solution of the Cauchy problem to this hierarchy of nonlinear evolution equations describes the processes of the creation and the propagation of correlations in many-particle quantum systems. Moreover, we consider the problem of the rigorous description of collective behavior of many-particle quantum systems by means of a one-particle (marginal) correlation operator that is a solution of the generalized quantum kinetic equation with initial correlations, in particular, correlations characterizing the condensed states of systems.
\end{abstract}

Key words: von Neumann hierarchy, nonlinear quantum BBGKY hierarchy, quantum kinetic equation, correlation of states, scaling limit.

2000 Mathematics Subject Classification: 35Q40; 47D06.

\section{Introduction}

In this review we consider mathematical problems concerning the description of processes of a creation and a propagation of correlations in quantum many-particle systems, namely, correlations in quantum systems both finitely and infinitely many particles in the condensed states and also the description of correlations by means of the state of typical particle of large quantum particle system.

As known, the marginal correlation operators give an equivalent approach to the description of the evolution of states of quantum systems of many particles in comparison with marginal density operators [1]. The physical interpretation of marginal correlation operators is that on the microscopic scale the macroscopic characteristics of fluctuations of mean values of observables of a system are determined by them [1], 22.

Traditionally marginal correlation operators are introduced by means of the cluster expansions of the marginal density operators governed by the BBGKY hierarchy [2]- [4]. In articles [5], [6] it was developed an approach based on the definition of the marginal correlation operators within 
the framework of dynamics of correlations governed by the von Neumann hierarchy. As a result of which it is established that the marginal correlation operators are governed by the hierarchy of nonlinear evolution equations, known as the quantum nonlinear BBGKY hierarchy (BogolyubovBorn-Green-Kirkwood-Yvon hierarchy), and its solution is represented in the form of series, the generating operator of every term of which are the corresponding-order cumulant of groups of nonlinear operators of the von Neumann hierarchy for correlation operators [7].

In the paper we also consider the problem of the rigorous description of the evolution of correlations in quantum many-particle systems by means of a one-particle (marginal) density operator that is a solution of the generalized quantum kinetic equation with initial correlations [9]. We remark that initial states specified by correlations are typical for the condensed states of manyparticle systems in contrast to their gaseous state [1], [10].

We note that in modern researches [11] [19], the conventional approach to the problem the rigorous derivation of kinetic equations lies in the construction of various scaling limits of a solution of equations, describing the evolution of the state of many-particle systems [20], in particular, of a mean field limit of a perturbative solution of the BBGKY hierarchy for a sequence of marginal density operators.

\section{Dynamics of quantum correlations}

As known [1], [2], quantum systems are described in terms of observables and states. The functional of the mean value of observables defines a duality between observables and states and as a consequence there exist two approaches to the description of the evolution of quantum systems, namely, in terms of observables that are governed by the Heisenberg equation and in terms of states governed by the von Neumann equation for the density operator, respectively. An equivalent approach to the description of states of quantum systems is given by means of operators determined by the cluster expansions of the density operator which are interpreted as correlation operators. In this section we consider fundamental equations describing the evolution of correlations of quantum systems with a finite number of particles.

\subsection{Preliminaries}

We denote by $\mathcal{F}_{\mathcal{H}}=\bigoplus_{n=0}^{\infty} \mathcal{H}^{\otimes n}$ the Fock space over the Hilbert space $\mathcal{H}$, where $\mathcal{H}^{\otimes n} \equiv \mathcal{H}_{n}$ is the $n$-particle Hilbert space. Let $\mathfrak{L}^{1}\left(\mathcal{H}_{n}\right)$ be the space of trace class operators $f_{n} \equiv f_{n}(1, \ldots, n) \in$ $\mathfrak{L}^{1}\left(\mathcal{H}_{n}\right)$ that satisfy the symmetry condition $f_{n}(1, \ldots, n)=f_{n}\left(i_{1}, \ldots, i_{n}\right)$ for arbitrary $\left(i_{1}, \ldots, i_{n}\right) \in$ $(1, \ldots, n)$, and equipped with the norm

$$
\left\|f_{n}\right\|_{\mathfrak{L}^{1}\left(\mathcal{H}_{n}\right)}=\operatorname{Tr}_{1, \ldots, n}\left|f_{n}(1, \ldots, n)\right|
$$

where $\operatorname{Tr}_{1, \ldots, n}$ are partial traces over $1, \ldots, n$ particles. We denote by $\mathfrak{L}_{0}^{1}\left(\mathcal{H}_{n}\right)$ the everywhere dense set of finite sequences of degenerate operators with infinitely differentiable kernels with compact supports.

On the space of trace class operators $\mathfrak{L}^{1}\left(\mathcal{H}_{n}\right)$ it is defined the one-parameter mapping

$$
\mathbb{R}^{1} \ni t \mapsto \mathcal{G}_{n}^{*}(t) f_{n} \doteq e^{-i t H_{n}} f_{n} e^{i t H_{n}},
$$


where the following units are used: $m=1$ is the mass of a particle, $h=2 \pi \hbar=1$ is a Planck constant, and the self adjoint operator $H_{n}$ is the Hamiltonian of $n$ particles, obeying MaxwellBoltzmann statistics. Further an inverse group to group (1) we will denote by $\left(\mathcal{G}_{n}^{*}\right)^{-1}(t)=\mathcal{G}_{n}^{*}(-t)$.

On its domain of the definition the infinitesimal generator $\mathcal{N}_{n}^{*}$ of the group of operators (1) is determined in the sense of the strong convergence of the space $\mathfrak{L}^{1}\left(\mathcal{H}_{n}\right)$ by the operator

$$
\lim _{t \rightarrow 0} \frac{1}{t}\left(\mathcal{G}_{n}^{*}(t) f_{n}-f_{n}\right)=-i\left(H_{n} f_{n}-f_{n} H_{n}\right) \doteq \mathcal{N}_{n}^{*} f_{n}
$$

that has the following structure: $\mathcal{N}_{n}^{*}=\sum_{j=1}^{n} \mathcal{N}^{*}(j)+\epsilon \sum_{j_{1}<j_{2}=1}^{n} \mathcal{N}_{\text {int }}^{*}\left(j_{1}, j_{2}\right)$, where the operator $\mathcal{N}^{*}(j)$ is a free motion generator of the von Neumann equation (the dual operator to the generator of the Heisenberg equation for observables) [2], the operator $\mathcal{N}_{\text {int }}^{*}$ is defined by means of the operator of a two-body interaction potential $\Phi$ by the formula: $\mathcal{N}_{\text {int }}^{*}\left(j_{1}, j_{2}\right) f_{n} \doteq-i\left(\Phi\left(j_{1}, j_{2}\right) f_{n}-\right.$ $\left.f_{n} \Phi\left(j_{1}, j_{2}\right)\right)$, and we denoted a scaling parameter by $\epsilon>0$.

Let the symbol $\sum_{\mathrm{P}:(1, \ldots, s)=\cup_{j} X_{j}}$ denote the sum over all possible partitions $\mathrm{P}$ of the set $(1, \ldots, s)$ into $|\mathrm{P}|$ nonempty mutually disjoint subsets $X_{j}$ and the set $\left(\left\{X_{1}\right\}, \ldots,\left\{X_{|\mathrm{P}|}\right\}\right)$ consists from elements which are subsets $X_{j} \subset(1, \ldots, s)$ of the set $(1, \ldots, s)$, i.e. $\left|\left(\left\{X_{1}\right\}, \ldots,\left\{X_{|\mathrm{P}|}\right\}\right)\right|=|\mathrm{P}|$. On the space $\mathfrak{L}^{1}\left(\mathcal{F}_{\mathcal{H}}\right)=\oplus_{n=0}^{\infty} \mathfrak{L}^{1}\left(\mathcal{H}_{n}\right)$ of sequences $f=\left(f_{0}, f_{1}, \ldots, f_{n}, \ldots\right)$ of trace class operators $f_{n} \in \mathfrak{L}^{1}\left(\mathcal{H}_{n}\right)$ and $f_{0} \in \mathbb{C}$ it is defined the following nonlinear one-parameter mapping

$$
\mathcal{G}(t ; 1, \ldots, s \mid f) \doteq \sum_{\mathrm{P}:(1, \ldots, s)=\cup_{j} X_{j}} \mathfrak{A}_{|\mathrm{P}|}\left(t,\left\{X_{1}\right\}, \ldots,\left\{X_{|\mathrm{P}|}\right\}\right) \prod_{X_{j} \subset \mathrm{P}} f_{\left|X_{j}\right|}\left(X_{j}\right), \quad s \geq 1,
$$

where the generating operator $\mathfrak{A}_{|\mathrm{P}|}(t)$ of this expansion is the $|\mathrm{P}|$ th-order cumulant of the groups of operators (1) defined by the following expansion [2]

$$
\mathfrak{A}_{|\mathrm{P}|}\left(t,\left\{X_{1}\right\}, \ldots,\left\{X_{|\mathrm{P}|}\right\}\right) \doteq \sum_{\mathrm{P}^{\prime}:\left(\left\{X_{1}\right\}, \ldots,\left\{X_{|\mathrm{P}|}\right\}\right)=\bigcup_{k} Z_{k}}(-1)^{\left|\mathrm{P}^{\prime}\right|-1}\left(\left|\mathrm{P}^{\prime}\right|-1\right) ! \prod_{Z_{k} \subset \mathrm{P}^{\prime}} \mathcal{G}_{\left|\theta\left(Z_{k}\right)\right|}^{*}\left(t, \theta\left(Z_{k}\right)\right),
$$

and $\theta$ is the declusterization mapping, namely, $\theta\left(\left\{X_{1}\right\}, \ldots,\left\{X_{|\mathrm{P}|}\right\}\right) \doteq(1, \ldots, s)$.

Below we adduce the examples of mapping expansions (3):

$$
\begin{aligned}
& \mathcal{G}(t ; 1 \mid f)=\mathfrak{A}_{1}(t, 1) f_{1}(1), \\
& \mathcal{G}(t ; 1,2 \mid f)=\mathfrak{A}_{1}(t,\{1,2\}) f_{2}(1,2)+\mathfrak{A}_{1+1}(t, 1,2) f_{1}(1) f_{1}(2), \\
& \mathcal{G}(t ; 1,2,3 \mid f)=\mathfrak{A}_{1}(t,\{1,2,3\}) f_{3}(1,2,3)+\mathfrak{A}_{1+1}(t, 1,\{2,3\}) f_{1}(1) f_{2}(2,3)+ \\
& \mathfrak{A}_{1+1}(t, 2,\{1,3\}) f_{1}(2) f_{2}(1,3)+\mathfrak{A}_{1+1}(t, 3,\{1,2\}) f_{1}(3) f_{2}(1,2)+ \\
& \mathfrak{A}_{3}(t, 1,2,3) f_{1}(1) f_{1}(2) f_{1}(3) .
\end{aligned}
$$

For $f_{s} \in \mathfrak{L}^{1}\left(\mathcal{H}_{s}\right), s \geq 1$, the mapping $\mathcal{G}(t ; 1, \ldots, s \mid f)$ is defined and, according to the inequality

$$
\left\|\mathfrak{A}_{|\mathrm{P}|}\left(t,\left\{X_{1}\right\}, \ldots,\left\{X_{|\mathrm{P}|}\right\}\right) f_{s}\right\|_{\mathfrak{L}^{1}\left(\mathcal{H}_{s}\right)} \leq|\mathrm{P}| ! e^{|\mathrm{P}|}\left\|f_{s}\right\|_{\mathfrak{L}^{1}\left(\mathcal{H}_{s}\right)}
$$

the following estimate is true

$$
\|\mathcal{G}(t ; 1, \ldots, s \mid f)\|_{\mathfrak{L}^{1}\left(\mathcal{H}_{s}\right)} \leq s ! e^{2 s} c^{s},
$$

where $c \equiv e^{3} \max \left(1, \max _{\mathrm{P}:(1, \ldots, s)=\bigcup_{i} X_{i}}\left\|f_{\left|X_{i}\right|}\right\|_{\mathfrak{L}^{1}\left(\mathcal{H}_{\left|X_{i}\right|}\right)}\right)$. On the space $\mathfrak{L}^{1}\left(\mathcal{F}_{\mathcal{H}}\right)$ one-parameter mapping (3) is a bounded strong continuous group of nonlinear operators. 


\subsection{The von Neumann hierarchy for correlation operators}

The evolution of all possible states of a quantum system of non-fixed, i.e. arbitrary but finite, number of identical particles, obeying the Maxwell-Boltzmann statistics, can be described by means of the sequence $g(t)=\left(g_{0}, g_{1}(t), \ldots, g_{s}(t), \ldots\right) \in \mathfrak{L}^{1}\left(\mathcal{F}_{\mathcal{H}}\right)$ of the correlation operators $g_{s}(t)=$ $g_{s}(t, 1, \ldots, s), s \geq 1$, governed by the Cauchy problem of the von Neumann hierarchy [5]:

$$
\begin{aligned}
& \frac{\partial}{\partial t} g_{s}(t, 1, \ldots, s)=\mathcal{N}_{s}^{*} g_{s}(t, 1, \ldots, s)+ \\
& \epsilon \sum_{\mathrm{P}:(1, \ldots, s)=X_{1} \cup X_{2}} \sum_{i_{1} \in X_{1}} \sum_{i_{2} \in X_{2}} \mathcal{N}_{\mathrm{int}}^{*}\left(i_{1}, i_{2}\right) g_{\left|X_{1}\right|}\left(t, X_{1}\right) g_{\left|X_{2}\right|}\left(t, X_{2}\right), \\
& \left.g_{s}(t)\right|_{t=0}=g_{s}^{0, \epsilon}, \quad s \geq 1
\end{aligned}
$$

where $\epsilon>0$ is a scaling parameter, the symbol $\sum_{\mathrm{P}:(1, \ldots, s)=X_{1} \cup X_{2}}$ means the sum over all possible partitions $\mathrm{P}$ of the set $(1, \ldots, s)$ into two nonempty mutually disjoint subsets $X_{1}$ and $X_{2}$, and the operator $\mathcal{N}_{s}^{*}$ is defined on the subspace $\mathfrak{L}_{0}^{1}\left(\mathcal{H}_{s}\right)$ by formula $(2)$.

We remark that correlation operators can be introduced by means of the cluster expansions of the density operators [2] (the kernel of a density operator is known as a density matrix) governed by a sequence of the von Neumann equations, and hence, they describe of the evolution of states by an equivalent method in comparison with the density operators. For quantum systems of fixed number of particles the state is described by finite sequence of correlation operators governed by a corresponding system of the von Neumann equations (6).

A solution (nonperturbative solution) of the Cauchy problem of the von Neumann hierarchy for correlation operators (6), (7) is represented by group of nonlinear operators (3)

$$
g(t, 1, \ldots, s)=\mathcal{G}(t ; 1, \ldots, s \mid g(0)), \quad s \geq 1,
$$

where a sequence of initial correlation operators (7) we denote by $g(0)=\left(g_{0}, g_{1}^{0, \epsilon}, \ldots, g_{n}^{0, \epsilon}, \ldots\right)$ and $g_{0} \in \mathbb{C}$.

We remark, if at initial time there are no correlations between particles, i.e. in case of initial states, satisfying a chaos condition [2], a sequence of initial correlation operators takes the form $g(0)=\left(0, g_{1}^{0, \epsilon}, 0, \ldots, 0, \ldots\right)$. Then solution (8) of the Cauchy problem of the von Neumann hierarchy (6), (7) is represented by the following expansions:

$$
g_{s}(t, 1, \ldots, s)=\mathfrak{A}_{s}(t, 1, \ldots, s) \prod_{i=1}^{s} g_{1}^{0, \epsilon}(i), \quad s \geq 1
$$

where the operator $\mathfrak{A}_{s}(t)$ is the sth-order cumulant of groups of operators (1) determined by the expansion

$$
\mathfrak{A}_{s}(t, 1, \ldots, s)=\sum_{\mathrm{P}:(1, \ldots, s)=\bigcup_{i} X_{i}}(-1)^{|\mathrm{P}|-1}(|\mathrm{P}|-1) ! \prod_{X_{i} \subset \mathrm{P}} \mathcal{G}_{\left|X_{i}\right|}^{*}\left(t, X_{i}\right),
$$

and we used notations accepted in formula (3).

We remark also that nonperturbative solution (8) of the Cauchy problem of the von Neumann hierarchy (6), (7) can be transformed to the perturbation (iteration) expansion as a result of the application of analogs of the Duhamel equation to cumulants (4) of groups of operators (1). 
The following statement is true [6]. In case of bounded interaction potentials for $t \in \mathbb{R}$ a solution of the Cauchy problem of the von Neumann hierarchy (6), (7) is determined by a sequence of correlation operators represented by formula (8). If $g_{n}^{0, \epsilon} \in \mathfrak{L}_{0}^{1}\left(\mathcal{H}_{n}\right) \subset \mathfrak{L}^{1}\left(\mathcal{H}_{n}\right)$, it is a strong solution and for arbitrary initial data $g_{n}^{0, \epsilon} \in \mathfrak{L}^{1}\left(\mathcal{H}_{n}\right)$ it is a weak solution.

The stated above results can be extended to quantum systems of bosons and fermions like in paper [6].

\section{The evolution of correlations in large quantum particle systems}

An equivalent approach to describing the states of quantum systems of many particles consists in describing states by means of marginal density operators governed by the BBGKI hierarchy or by means of operators determined by their cluster expansions, which are interpreted as marginal correlation operators [1]. On the microscopic scale the macroscopic characteristics of fluctuations of observables are directly determined by the marginal correlation operators. Such approach allows us to describe the evolution of correlations in quantum systems both with finite and infinite number of particles.

\subsection{The hierarchy of evolution equations for marginal correlation op- erators}

Traditionally marginal correlation operators are determined by means of the cluster expansions of the marginal density operators [2]- [4]. We introduce the marginal correlation operators in the framework of the solution of the Cauchy problem for the von Neumann hierarchy (6), (7) by the following series expansions:

$$
G_{s}(t, 1, \ldots, s) \doteq \sum_{n=0}^{\infty} \frac{1}{n !} \operatorname{Tr}_{s+1, \ldots, s+n} \mathcal{G}(t ; 1, \ldots, s+n \mid g(0)), \quad s \geq 1 .
$$

According to estimate (5), series 10 exists and the following estimate holds: $\left\|G_{s}(t)\right\|_{\mathfrak{L}^{1}\left(\mathcal{H}_{s}\right)} \leq$ $s !\left(2 e^{2}\right)^{s} c^{s} \sum_{n=0}^{\infty}\left(2 e^{2}\right)^{n} c^{n}$, where $c \equiv e^{3} \max \left(1, \max _{\mathrm{P}:(1, \ldots, s)=\bigcup_{i} X_{i}}\left\|g_{\left|X_{i}\right|}(0)\right\|_{\mathfrak{L}^{1}\left(\mathcal{H}_{\left|X_{i}\right|}\right)}\right)$.

We remark that the macroscopic characteristics of fluctuations of observables are directly determined by marginal correlation operators (10), for example, the functional of the dispersion of the additive-type observables, i.e. $A^{(1)}=\left(0, a_{1}(1), \ldots, \sum_{i_{1}=1}^{n} a_{1}\left(i_{1}\right), \ldots\right)$, is represented by the formula 1

$$
\left\langle\left(A^{(1)}-\left\langle A^{(1)}\right\rangle\right)^{2}\right\rangle(t)=\operatorname{Tr}_{1}\left(a_{1}^{2}(1)-\left\langle A^{(1)}\right\rangle^{2}(t)\right) G_{1}(t, 1)+\operatorname{Tr}_{1,2} a_{1}(1) a_{1}(2) G_{2}(t, 1,2),
$$

where $\left\langle A^{(1)}\right\rangle(t)=\operatorname{Tr}_{1} a_{1}(1) G_{1}(t, 1)$ is a mean-value functional of the additive-type observable [2].

Then the evolution of all possible states of large quantum particle systems, obeying the MaxwellBoltzmann statistics, can be described by means of the sequence $G(t)=\left(I, G_{1}(t), G_{2}(t), \ldots, G_{s}(t)\right.$, $\ldots) \in \mathfrak{L}^{1}\left(\mathcal{F}_{\mathcal{H}}\right)$ of marginal correlation operators governed by the Cauchy problem of the following 
hierarchy of nonlinear evolution equations (the nonlinear quantum BBGKY hierarchy):

$$
\begin{aligned}
& \frac{\partial}{\partial t} G_{s}(t, 1, \ldots, s)=\mathcal{N}_{s}^{*} G_{s}(t, 1, \ldots, s)+ \\
& \left.\epsilon \sum_{\mathrm{P}:(1, \ldots, s)=X_{1} \cup X_{2}} \sum_{i_{1} \in X_{1}} \sum_{i_{2} \in X_{2}} \mathcal{N}_{\text {int }}^{*}\left(i_{1}, i_{2}\right) G_{\left|X_{1}\right|}\left(t, X_{1}\right) G_{\left|X_{2}\right|}\left(t, X_{2}\right)\right)+ \\
& \quad \operatorname{Tr}_{s+1} \sum_{\substack{i \in Y \\
\mathcal{N}_{\text {int }}^{*}}}^{*}(i, s+1)\left(G_{s+1}(t, 1, \ldots, s+1)+\right. \\
& \left.\quad \sum_{\substack{(1, \ldots, s+1)=X_{1} \cup X_{2}, i \in X_{1} ; s+1 \in X_{2}}} G_{\left|X_{1}\right|}\left(t, X_{1}\right) G_{\left|X_{2}\right|}\left(t, X_{2}\right)\right), \\
& \left.G_{s}(t)\right|_{t=0}=G_{s}^{0, \epsilon}, \quad s \geq 1,
\end{aligned}
$$

where $\epsilon>0$ is a scaling parameter and we use accepted in hierarchy (6) notations.

If $G(0)=\left(I, G_{1}^{0, \epsilon}(1), \ldots, G_{s}^{0, \epsilon}(1, \ldots, s), \ldots\right)$ is a sequence of initial marginal correlation operators (12), then a nonperturbative solution of the Cauchy problem (11), (12) is represented by the following sequence of self-adjoint operators:

$$
G_{s}(t, 1, \ldots, s)=\sum_{n=0}^{\infty} \frac{1}{n !} \operatorname{Tr}_{s+1, \ldots, s+n} \mathfrak{A}_{1+n}(t ;\{1, \ldots, s\}, s+1, \ldots, s+n \mid G(0)), \quad s \geq 1,
$$

where the generating operator $\mathfrak{A}_{1+n}(t ;\{1, \ldots, s\}, s+1, \ldots, s+n \mid G(0))$ of this series is the $(1+n) t h$-order cumulant of groups of nonlinear operators (3):

$$
\begin{aligned}
& \mathfrak{A}_{1+n}(t ;\{1, \ldots, s\}, s+1, \ldots, s+n \mid G(0)) \doteq \\
& \quad \sum_{\mathrm{P}:(\{1, \ldots, s\}, s+1, \ldots, s+n)=\cup_{k} X_{k}}(-1)^{|\mathrm{P}|-1}(|\mathrm{P}|-1) ! \mathcal{G}\left(t ; \theta\left(X_{1}\right) \mid \ldots \mathcal{G}\left(t ; \theta\left(X_{|\mathrm{P}|}\right) \mid G(0)\right) \ldots\right), \quad n \geq 0,
\end{aligned}
$$

and composition of mappings (3) of the corresponding noninteracting groups of particles we denoted by $\mathcal{G}\left(t ; \theta\left(X_{1}\right) \mid \ldots \mathcal{G}\left(t ; \theta\left(X_{|\mathrm{P}|}\right) \mid G(0)\right) \ldots\right)$, for example,

$$
\begin{aligned}
& \mathcal{G}(t ; 1 \mid \mathcal{G}(t ; 2 \mid f))=\mathfrak{A}_{1}(t, 1) \mathfrak{A}_{1}(t, 2) f_{2}(1,2), \\
& \mathcal{G}(t ; 1,2 \mid \mathcal{G}(t ; 3 \mid f))=\mathfrak{A}_{1}(t,\{1,2\}) \mathfrak{A}_{1}(t, 3) f_{3}(1,2,3)+ \\
& \mathfrak{A}_{2}(t, 1,2) \mathfrak{A}_{1}(t, 3)\left(f_{1}(1) f_{2}(2,3)+f_{1}(2) f_{2}(1,3)\right) .
\end{aligned}
$$

Below we adduce the examples of expansions (14). The first order cumulant of the groups of nonlinear operators (3) is the same group of nonlinear operators, i.e.

$$
\mathfrak{A}_{1}(t ;\{1, \ldots, s\} \mid G(0))=\mathcal{G}(t ; 1, \ldots, s \mid G(0)) .
$$

In case of $s=2$ the second order cumulant of nonlinear operators (3) has the structure

$$
\begin{aligned}
& \mathfrak{A}_{1+1}(t ;\{1,2\}, 3 \mid G(0))=\mathcal{G}(t ; 1,2,3 \mid G(0))-\mathcal{G}(t ; 1,2 \mid \mathcal{G}(t ; 3 \mid G(0)))= \\
& \mathfrak{A}_{1+1}(t,\{1,2\}, 3) G_{3}^{0, \epsilon}(1,2,3)+\left(\mathfrak{A}_{1+1}(t,\{1,2\}, 3)-\mathfrak{A}_{1+1}(t, 2,3) \mathfrak{A}_{1}(t, 1)\right) G_{1}^{0, \epsilon}(1) G_{2}^{0, \epsilon}(2,3)+ \\
& \left(\mathfrak{A}_{1+1}(t,\{1,2\}, 3)-\mathfrak{A}_{1+1}(t, 1,3) \mathfrak{A}_{1}(t, 2)\right) G_{1}^{0, \epsilon}(2) G_{2}^{0, \epsilon}(1,3)+ \\
& \mathfrak{A}_{1+1}(t,\{1,2\}, 3) G_{1}^{0, \epsilon}(3) G_{2}^{0, \epsilon}(1,2)+\mathfrak{A}_{3}(t, 1,2,3) G_{1}^{0, \epsilon}(1) G_{1}^{0, \epsilon}(2) G_{1}^{0, \epsilon}(3),
\end{aligned}
$$


where the operator

$$
\mathfrak{A}_{3}(t, 1,2,3)=\mathfrak{A}_{1+1}(t,\{1,2\}, 3)-\mathfrak{A}_{1+1}(t, 2,3) \mathfrak{A}_{1}(t, 1)-\mathfrak{A}_{1+1}(t, 1,3) \mathfrak{A}_{1}(t, 2)
$$

is the third order cumulant (9) of groups of operators (1).

In case of initial data specified by the sequence of marginal correlation operators

$$
G^{(c)}=\left(0, G_{1}^{0, \epsilon}, 0, \ldots, 0, \ldots\right)
$$

i.e. initial states satisfying a chaos property [20], according to definition (14), marginal correlation operators (13) are represented by the following series expansions:

$$
G_{s}(t, 1, \ldots, s)=\sum_{n=0}^{\infty} \frac{1}{n !} \operatorname{Tr}_{s+1, \ldots, s+n} \mathfrak{A}_{s+n}(t ; 1, \ldots, s+n) \prod_{i=1}^{s+n} G_{1}^{0, \epsilon}(i), \quad s \geq 1,
$$

where the generating operator $\mathfrak{A}_{s+n}(t)$ is $(s+n)$ th-order cumulant (9) of groups of operators (1).

We note that within the framework of the description of states by means of marginal density operators defined by cluster expansions over marginal correlation operators:

$$
F_{s}^{0, \epsilon}(1, \ldots, s)=\sum_{\mathrm{P}:(1, \ldots, s)=\cup_{i} X_{i}} \prod_{X_{i} \subset \mathrm{P}} G_{\left|X_{i}\right|}^{0, \epsilon}\left(X_{i}\right), \quad s \geq 1
$$

initial states described like to sequence $\sqrt{15})$ is specified by the sequence $F^{(c)}=\left(I, F_{1}^{0, \epsilon}(1), \ldots\right.$, $\left.\prod_{i=1}^{n} F_{1}^{0, \epsilon}(i), \ldots\right)$, and in case of sequence (16) the marginal density operators are represented by the following series expansions (a nonperturbative solution of the quantum BBGKY hierarchy [2]):

$$
F_{s}(t, 1, \ldots, s)=\sum_{n=0}^{\infty} \frac{1}{n !} \operatorname{Tr}_{s+1, \ldots, s+n} \mathfrak{A}_{1+n}(t ;\{1, \ldots, s\}, s+1, \ldots, s+n) \prod_{i=1}^{s+n} F_{1}^{0, \epsilon}(i), \quad s \geq 1,
$$

where the generating operator $\mathfrak{A}_{1+n}(t)$ is the $(1+n)$ th-order cumulant of groups of operators (1).

One of the possible methods to derive series expansion (13) for the marginal correlation operators lies in the substitution of the cluster expansions of groups of nonlinear operators (3) over cumulants (14) and the sequence of initial correlation operators $g(0)=\left(I, g_{1}^{0, \epsilon}(1), \ldots, g_{n}^{0, \epsilon}(1, \ldots, n), \ldots\right)$ determined by means of the marginal correlation operators:

$$
g_{s}^{0, \epsilon}(1, \ldots, s) \doteq \sum_{n=0}^{\infty}(-1)^{n} \frac{1}{n !} \operatorname{Tr}_{s+1, \ldots, s+n} G_{s+n}^{0, \epsilon}(1, \ldots, s+n), \quad s \geq 1,
$$

into the definition of marginal correlation operators 10. Indeed, developing the generating operators of series 13 as the following cluster expansions:

$$
\mathcal{G}(t ; 1, \ldots, s+n \mid f)=\sum_{\mathrm{P}:(1, \ldots, s+n)=\cup_{k} X_{k}} \mathfrak{A}_{\left|X_{1}\right|}\left(t ; X_{1} \mid \ldots \mathfrak{A}_{\left|X_{|\mathrm{P}|}\right|}\left(t ; X_{|\mathrm{P}|} \mid f\right) \ldots\right), \quad n \geq 0
$$

according to definition (17), we derive expressions (13). The solutions of recursive relations (18) are represented by expansions 14 . 
We remark that on the space $\mathfrak{L}^{1}\left(\mathcal{F}_{\mathcal{H}}\right)$ the generating operator 114 ) of series expansion $(13)$ can be represented as the $(1+n)$ th-order reduced cumulant of the groups of nonlinear operators (3) of the von Neumann hierarchy [2]:

$$
\begin{aligned}
& U_{1+n}(t ;\{1, \ldots, s\}, s+1, \ldots, s+n \mid G(0)) \doteq \\
& \sum_{k=0}^{n}(-1)^{k} \frac{n !}{k !(n-k) !} \sum_{\mathrm{P}:(\theta(\{1, \ldots, s\}), s+1, \ldots, s+n-k)=\bigcup_{i} X_{i}} \mathfrak{A}_{|\mathrm{P}|}\left(t,\left\{X_{1}\right\}, \ldots,\left\{X_{|\mathrm{P}|}\right\}\right) \\
& \sum_{k_{1}=0}^{k} \frac{k !}{k_{1} !\left(k-k_{1}\right) !} \cdots \sum_{k_{|\mathrm{P}|-1}=0}^{k_{|\mathrm{P}|-2}} \frac{k_{|\mathrm{P}|-2} !}{k_{|\mathrm{P}|-1} !\left(k_{|\mathrm{P}|-2}-k_{|\mathrm{P}|-1}\right) !} G_{\left|X_{1}\right|+k-k_{1}}^{0, \epsilon} \\
& \left.s+n-k+1, \ldots, s+n-k_{1}\right) \ldots G_{\left|X_{|\mathrm{P}|}\right|+k_{|\mathrm{P}|-1}}^{0, \epsilon}, \\
& \left.s+X_{|\mathrm{P}|}, s+n-k_{|\mathrm{P}|-1}+1, \ldots, s+n\right), \quad n \geq 0
\end{aligned}
$$

as examples, we adduce the simplest examples of reduced cumulants (19):

$$
\begin{aligned}
& U_{1}(t ;\{1, \ldots, s\} \mid G(0))=\mathcal{G}(t ; 1, \ldots, s \mid G(0))= \\
& \quad \sum_{\mathrm{P}:(1, \ldots, s)=\bigcup_{i} X_{i}} \mathfrak{A}_{|\mathrm{P}|}\left(t,\left\{X_{1}\right\}, \ldots,\left\{X_{|\mathrm{P}|}\right\}\right) \\
& U_{1+1}(t ;\{1, \ldots, s\}, s+1 \mid G(0))=\sum_{X_{i} \subset \mathrm{P}} G_{\left|X_{i}\right|}^{0, \epsilon}\left(X_{i}\right), \\
& \quad \sum_{\mathrm{P}:(1, \ldots, s+1)=\bigcup_{i} X_{i}} \mathfrak{A}_{|\mathrm{P}|}\left(t,\left\{X_{1}\right\}, \ldots,\left\{X_{|\mathrm{P}|}\right\}\right) \prod_{X_{i} \subset \mathrm{P}} G_{\left|X_{i}\right|}^{0, \epsilon}\left(X_{i}\right)- \\
& \mathfrak{P}_{|\mathrm{P}|}\left(t,\left\{X_{1}\right\}, \ldots,\left\{X_{|\mathrm{P}|}\right\}\right) \sum_{j=1}^{|\mathrm{P}|} G_{\left|X_{j}\right|+1}^{0, \epsilon}\left(X_{j}, s+1\right) \prod_{\substack{X_{i} \subset \mathrm{P} \\
X_{i} \neq X_{j}}} G_{\left|X_{i}\right|}^{0, \epsilon}\left(X_{i}\right) .
\end{aligned}
$$

We note also that a nonperturbative solution of the nonlinear quantum BBGKY hierarchy (13) or in the form of series expansions with generating operators (19) can be transformed to the perturbation (iteration) series as a result of the application of analogs of the Duhamel equation to cumulants (4) of groups of operators (1).

The following statement is true [7]. If $\max _{n \geq 1}\left\|G_{n}^{0, \epsilon}\right\|_{\mathfrak{L}^{1}\left(\mathcal{H}_{n}\right)}<\left(2 e^{3}\right)^{-1}$, then in case of bounded interaction potentials for $t \in \mathbb{R}$ a solution of the Cauchy problem of the nonlinear quantum BBGKY hierarchy (11), 12 is determined by a sequence of marginal correlation operators represented by series expansions (13). If $G_{n}^{0, \epsilon} \in \mathfrak{L}_{0}^{1}\left(\mathcal{H}_{n}\right) \subset \mathfrak{L}^{1}\left(\mathcal{H}_{n}\right)$, it is a strong solution and for arbitrary initial data $G_{n}^{0, \epsilon} \in \mathfrak{L}^{1}\left(\mathcal{H}_{n}\right)$ it is a weak solution.

\subsection{A mean field asymptotic behavior of marginal correlation opera- tors}

Now we deals with a scaling asymptotic behavior of the constructed marginal correlation operators in a mean field limit in case of initial states satisfied condition (15).

Let us observe that, if $f_{s} \in \mathfrak{L}^{1}\left(\mathcal{H}_{s}\right)$, then for arbitrary finite time interval for an asymptotically perturbed first-order cumulant (9) of the groups of operators (1), i.e. for the strongly continuous group (1), the following equality is valid

$$
\lim _{\epsilon \rightarrow 0}\left\|\mathcal{G}_{s}^{*}(t, 1, \ldots, s) f_{s}-\prod_{j=1}^{s} \mathcal{G}_{1}^{*}(t, j) f_{s}\right\|_{\mathfrak{L}^{1}\left(\mathcal{H}_{s}\right)}=0 .
$$


As a result of this for the $(s+n) t h$-order cumulants of asymptotically perturbed groups of operators (1) the following equalities are true:

$$
\lim _{\epsilon \rightarrow 0}\left\|\frac{1}{\epsilon^{n}} \mathfrak{A}_{s+n}(t, 1, \ldots, s+n) f_{s+n}\right\|_{\mathfrak{L}^{1}\left(\mathcal{H}_{s+n}\right)}=0, \quad s \geq 2 .
$$

We assume the existence of a mean field limit for initial marginal correlation operator (or a one-particle density operator) in the following sense

$$
\lim _{\epsilon \rightarrow 0}\left\|\epsilon G_{1}^{0, \epsilon}-g_{1}^{0}\right\|_{\mathfrak{L}^{1}(\mathcal{H})}=0 .
$$

Then, taking into account equality (20), and since the $n$th term of series expansion (16) for $s$-particle marginal correlation operator is determined by the $(s+n)$ th-order cumulant of asymptotically perturbed groups of operators (1), we establish the property of the propagation of initial chaos 15):

$$
\lim _{\epsilon \rightarrow 0}\left\|\epsilon^{s} G_{s}(t)\right\|_{\mathfrak{L}^{1}\left(\mathcal{H}_{s}\right)}=0, \quad s \geq 2 .
$$

If for the initial marginal correlation operator equality (21) holds, then in case of $s=1$ for series expansion (16) the following equality is true

$$
\lim _{\epsilon \rightarrow 0}\left\|\epsilon G_{1}(t)-g_{1}(t)\right\|_{\mathfrak{L}^{1}(\mathcal{H})}=0
$$

where for arbitrary finite time interval the limit one-particle marginal correlation operator $g_{1}(t, 1)$ is given by the norm convergent series on the space $\mathfrak{L}^{1}(\mathcal{H})$

$$
\begin{aligned}
& g_{1}(t, 1)=\sum_{n=0}^{\infty} \int_{0}^{t} d t_{1} \ldots \int_{0}^{t_{n-1}} d t_{n} \operatorname{Tr}_{2, \ldots, n+1} \mathcal{G}_{1}^{*}\left(t-t_{1}, 1\right) \mathcal{N}_{\text {int }}^{*}(1,2) \prod_{j_{1}=1}^{2} \mathcal{G}_{1}^{*}\left(t_{1}-t_{2}, j_{1}\right) \ldots \\
& \prod_{i_{n}=1}^{n} \mathcal{G}_{1}^{*}\left(t_{n}-t_{n}, i_{n}\right) \sum_{k_{n}=1}^{n} \mathcal{N}_{\text {int }}^{*}\left(k_{n}, n+1\right) \prod_{j_{n}=1}^{n+1} \mathcal{G}_{1}^{*}\left(t_{n}, j_{n}\right) \prod_{i=1}^{n+1} g_{1}^{0}(i) .
\end{aligned}
$$

In series expansion (23) the operator $\mathcal{N}_{\text {int }}^{*}\left(j_{1}, j_{2}\right)$ is defined by formula (2) and the group of operators $\mathcal{G}_{1}^{*}(t)$ is defined by (1). For bounded interaction potential series (23) is norm convergent on the space $\mathfrak{L}^{1}(\mathcal{H})$ under the condition that $t<t_{0} \equiv\left(2\|\Phi\|_{\mathfrak{L}\left(\mathcal{H}_{2}\right)}\left\|g_{1}^{0}\right\|_{\mathfrak{L}^{1}(\mathcal{H})}\right)^{-1}$.

As a result of differentiation in the sense of the norm convergence of the space $\mathfrak{L}^{1}(\mathcal{H})$ by the time variable of the operator represented by series expansion 23 we conclude that limit oneparticle marginal correlation operator (23) is governed by the Cauchy problem of the quantum Vlasov kinetic equation

$$
\begin{aligned}
& \frac{\partial}{\partial t} g_{1}(t, 1)=\mathcal{N}^{*}(1) g_{1}(t, 1)+\operatorname{Tr}_{2} \mathcal{N}_{\text {int }}^{*}(1,2) g_{1}(t, 1) g_{1}(t, 2), \\
& \left.g_{1}(t)\right|_{t=0}=g_{1}^{0} .
\end{aligned}
$$


Then for pure states we derive the Hartree equation [2], indeed, in terms of the kernel $g_{1}\left(t, q ; q^{\prime}\right)=$ $\psi(t, q) \psi\left(t, q^{\prime}\right)$ of operator (23), describing a pure state, quantum kinetic equation (24) is reduced to the Hartree equation

$$
i \frac{\partial}{\partial t} \psi(t, q)=-\frac{1}{2} \Delta_{q} \psi(t, q)+\int d q^{\prime} \Phi\left(q-q^{\prime}\right)\left|\psi\left(t, q^{\prime}\right)\right|^{2} \psi(t, q),
$$

where the function $\Phi$ is a two-body interaction potential.

We note that in case of pure states kinetic equation (24) can be reduced to the nonlinear Schrödinger equation [13] or to the Gross-Pitaevskii kinetic equation [14].

\section{The description of processes of a creation and a propa- gation of correlations by means of kinetic equations in the condensed states}

In this section we consider mathematical problems concerning the description of processes of creation and propagation of correlations within the framework of the state of typical particle of a quantum systems of many particles, in other words, an approach to the description of evolution of correlations by means of quantum kinetic equations is developing.

\subsection{Marginal correlation functionals of the state}

Further we shall consider the case of initial states specified by a one-particle marginal density operator with correlations, namely, initial states specified by the following sequence of marginal correlation operators:

$$
G^{(c)}=\left(I, G_{1}^{0, \epsilon}(1), g_{2}^{\epsilon}(1,2) \prod_{i=1}^{2} G_{1}^{0, \epsilon}(i), \ldots, g_{n}^{\epsilon}(1, \ldots, n) \prod_{i=1}^{n} G_{1}^{0, \epsilon}(i), \ldots\right)
$$

where the operators $g_{n}^{\epsilon}(1, \ldots, n) \equiv g_{n}^{\epsilon} \in \mathfrak{L}_{0}^{1}\left(\mathcal{H}_{n}\right), n \geq 2$, are specified the initial correlations. We remark that such assumption about initial states is intrinsic for the kinetic description of manyparticle systems. On the other hand, initial data (26) is typical for the condensed states of large quantum systems of particle, for example, the equilibrium state of the Bose condensate satisfies the weakening of correlation condition with the correlations which characterize the condensed state [1], [10].

For initial states specified in terms of a one-particle density operator and correlation operators (26) the evolution of states given within the framework of the sequence $G(t)=\left(I, G_{1}(t), \ldots\right.$, $\left.G_{s}(t), \ldots\right)$ of marginal correlation operators $(13)$ can be described by means of the sequence $G\left(t \mid G_{1}(t)\right)=\left(I, G_{1}(t), G_{2}\left(t \mid G_{1}(t)\right), \ldots, G_{s}\left(t \mid G_{1}(t)\right), \ldots\right)$ of marginal correlation functionals $G_{s}\left(t, 1, \ldots, s \mid G_{1}(t)\right), s \geq 2$, with respect to the one-particle correlation operator $G_{1}(t)$ governed by the kinetic equation [8], [9].

In the case under consideration the marginal correlation functionals $G_{s}\left(t \mid G_{1}(t)\right), s \geq 2$, are 
defined with respect to the one-particle (marginal) density operator

$$
\begin{aligned}
& G_{1}(t, 1)= \\
& \sum_{n=0}^{\infty} \frac{1}{n !} \operatorname{Tr}_{2, \ldots, 1+n} \mathfrak{A}_{1+n}(t, 1, \ldots, n+1) \sum_{\mathrm{P}:(1, \ldots, n+1)=\cup_{i} X_{i}} \prod_{X_{i} \subset \mathrm{P}} g_{\left|X_{i}\right|}^{\epsilon}\left(X_{i}\right) \prod_{i=1}^{n+1} G_{1}^{0, \epsilon}(i),
\end{aligned}
$$

where the generating operator $\mathfrak{A}_{1+n}(t)$ is the $(1+n)$ - th order cumulant (4) of the groups of operators (1), and these functionals are represented by the series expansions:

$$
\begin{aligned}
& G_{s}\left(t, 1, \ldots, s \mid G_{1}(t)\right)= \\
& \sum_{n=0}^{\infty} \frac{1}{n !} \operatorname{Tr}_{s+1, \ldots, s+n} \mathfrak{G}_{s+n}(t, \theta(\{1, \ldots, s\}), s+1, \ldots, s+n) \prod_{i=1}^{s+n} G_{1}(t, i), \quad s \geq 2,
\end{aligned}
$$

where the $(s+n) t h$-order generating operator $\mathfrak{G}_{s+n}(t), n \geq 0$, of this series is determined by the following expansion

$$
\begin{aligned}
& \mathfrak{G}_{s+n}(t, \theta(\{1, \ldots, s\}), s+1, \ldots, s+n)= \\
& n ! \sum_{k=0}^{n}(-1)^{k} \sum_{n_{1}=1}^{n} \ldots \sum_{n_{k}=1}^{n-n_{1}-\ldots-n_{k-1}} \frac{1}{\left(n-n_{1}-\ldots-n_{k}\right) !} \times \\
& \breve{\mathfrak{A}}_{s+n-n_{1}-\ldots-n_{k}}\left(t, \theta(\{1, \ldots, s\}), s+1, \ldots, s+n-n_{1}-\ldots-n_{k}\right) \times \\
& \prod_{j=1}^{k} \sum_{\substack{\mathrm{D}_{j}: Z_{j}=\bigcup_{l_{j}} X_{l_{j}},\left|\mathrm{D}_{j}\right| \leq s+n-n_{1}-\cdots-n_{j}}} \frac{1}{\left|\mathrm{D}_{j}\right| !} \sum_{i_{1} \neq \ldots \neq i_{\left|\mathrm{D}_{j}\right|} \mid=1}^{s+n-n_{1}-\ldots-n_{j}} \prod_{X_{l_{j}} \subset \mathrm{D}_{j}} \frac{1}{\left|X_{l_{j}}\right| !} \breve{\mathfrak{A}}_{1+\left|X_{l_{j}}\right|}\left(t, i_{l_{j}}, X_{l_{j}}\right) \text {. }
\end{aligned}
$$

In formula (29) the sum over all possible dissections [23] of the linearly ordered set $Z_{j} \equiv(s+n-$ $\left.n_{1}-\ldots-n_{j}+1, \ldots, s+n-n_{1}-\ldots-n_{j-1}\right)$ on no more than $s+n-n_{1}-\ldots-n_{j}$ linearly ordered subsets we denote by $\sum_{\mathrm{D}_{j}: Z_{j}=\cup_{l_{j}} X_{l_{j}}}$ and the $(s+n) t h$-order scattering cumulant is defined by the formula

$$
\breve{\mathfrak{A}}_{s+n}(t, \theta(\{1, \ldots, s\}), s+1, \ldots, s+n) \doteq \mathfrak{A}_{s+n}(t, 1, \ldots, s+n) g_{s+n}^{\epsilon}(1, \ldots, s+n) \prod_{i=1}^{s+n} \mathfrak{A}_{1}^{-1}(t, i),
$$

where the operator $g_{s+n}^{\epsilon}(1, \ldots, s+n)$ is specified initial correlations (26), and notations accepted above were used. If $\left\|G_{1}(t)\right\|_{\mathfrak{L}^{1}(\mathcal{H})}<e^{-(3 s+2)}$, then for arbitrary $t \in \mathbb{R}$ series expansion (28) converges in the norm of the space $\mathfrak{L}^{1}\left(\mathcal{H}_{s}\right)$.

We adduce simplest examples of generating operators (29):

$$
\begin{aligned}
& \left.\mathfrak{G}_{s}(t, \theta(\{1, \ldots, s\}))=\breve{\mathfrak{A}}_{s}(t, \theta(\{1, \ldots, s\}))=\mathfrak{A}_{s}(t, 1, \ldots, s)\right) g_{s}^{\epsilon}(1, \ldots, s) \prod_{i=1}^{s} \mathfrak{A}_{1}^{-1}(t, i), \\
& \mathfrak{G}_{s+1}(t, \theta(\{1, \ldots, s\}), s+1)=\mathfrak{A}_{s+1}(t, 1, \ldots, s+1) g_{s+1}^{\epsilon}(1, \ldots, s+1) \prod_{i=1}^{s+1} \mathfrak{A}_{1}^{-1}(t, i)- \\
& \mathfrak{A}_{s}(t, 1, \ldots, s) g_{s}^{\epsilon}(1, \ldots, s) \prod_{i=1}^{s} \mathfrak{A}_{1}^{-1}(t, i) \sum_{j=1}^{s} \mathfrak{A}_{2}(t, j, s+1) g_{2}^{\epsilon}(j, s+1) \mathfrak{A}_{1}^{-1}(t, j) \mathfrak{A}_{1}^{-1}(t, s+1) .
\end{aligned}
$$


A method of the construction of marginal correlation functionals $(28)$ is based on the application of kinetic cluster expansions [2] to the generating operators of series (13).

We emphasize that marginal correlation functionals 28 describe the all possible correlations generated by dynamics of large quantum particle system with initial correlations by means of a one-particle density operator.

\subsection{The generalized quantum kinetic equation with initial correlations}

Now we establish the evolution equation for one-particle (marginal) density operator (27). As a result of the differentiation over time variable of the operator represented by series expansion (27) in the sense of the norm convergence of the space $\mathfrak{L}^{1}(\mathcal{H})$, then due to the application of the kinetic cluster expansions [22] to the generating operators of obtained series expansion, for one-particle density operator (27) we derive the following identity:

$$
\begin{gathered}
\frac{\partial}{\partial t} G_{1}(t, 1)=\mathcal{N}^{*}(1) G_{1}(t, 1)+\epsilon \operatorname{Tr}_{2} \mathcal{N}_{\text {int }}^{*}(1,2) G_{1}(t, 1) G_{1}(t, 2)+ \\
+\epsilon \operatorname{Tr}_{2} \mathcal{N}_{\text {int }}^{*}(1,2) G_{2}\left(t, 1,2 \mid G_{1}(t)\right),
\end{gathered}
$$

where the second part of the collision integral in equality 30 is determined in terms of the marginal correlation functional represented by series expansions (28) in case of $s=2$. This identity we treat as the quantum kinetic equation and we refer to this evolution equation as the generalized quantum kinetic equation with initial correlations.

We emphasize that the coefficients in an expansion of the collision integral of the non-Markovian kinetic equation (30) are determined by the operators specified initial correlations (26).

On the space $\mathfrak{L}^{1}(\mathcal{H})$ for the Cauchy problem of the established generalized quantum kinetic equation with initial correlations the following statement is true 22$]$. If $\left\|G_{1}^{0, \epsilon}\right\|_{\mathfrak{L}^{1}(\mathcal{H})}<\left(e\left(1+e^{9}\right)\right)^{-1}$, a global in time solution of the Cauchy problem of kinetic equation 30 is determined by series expansion (27). For initial data $G_{1}^{0, \epsilon} \in \mathfrak{L}_{0}^{1}(\mathcal{H})$ it is a strong solution and for an arbitrary initial data it is a weak solution.

The proof of this existence statement is similar to the proof in the case of the generalized quantum kinetic equation given in [23].

\subsection{On a propagation of initial correlations in a mean field limit}

Further we establish a mean field asymptotic behavior of constructed marginal correlation functionals (28) in case of initial states specified by a one-particle density operator and correlation operators (26).

We assume the existence of a mean field limit of initial a one-particle density operator in sense (21) and for initial correlation operators as follows:

$$
\lim _{\epsilon \rightarrow 0}\left\|g_{n}^{\epsilon}-g_{n}\right\|_{\mathfrak{L}^{1}\left(\mathcal{H}_{n}\right)}=0, \quad n \geq 2 .
$$

Then in consequence of the validity of equalities (20) for one-particle density operator (27) the following statement is true [9]. If conditions (21) and (31) hold, then for series expansion (27) the equality holds

$$
\lim _{\epsilon \rightarrow 0}\left\|\epsilon G_{1}(t)-g_{1}(t)\right\|_{\mathfrak{L}^{1}(\mathcal{H})}=0
$$


where for finite time interval the limit one-particle density operator $g_{1}(t)$ is represented by the following norm convergent series on the space $\mathfrak{L}^{1}(\mathcal{H})$

$$
\begin{aligned}
& g_{1}(t, 1)=\sum_{n=0}^{\infty} \int_{0}^{t} d t_{1} \ldots \int_{0}^{t_{n-1}} d t_{n} \operatorname{Tr}_{2, \ldots, n+1} \mathcal{G}_{1}^{*}\left(t-t_{1}, 1\right) \mathcal{N}_{\mathrm{int}}^{*}(1,2) \prod_{j_{1}=1}^{2} \mathcal{G}_{1}^{*}\left(t_{1}-t_{2}, j_{1}\right) \ldots \\
& \prod_{i_{n}=1}^{n} \mathcal{G}_{1}^{*}\left(t_{n}-t_{n}, i_{n}\right) \sum_{k_{n}=1}^{n} \mathcal{N}_{\mathrm{int}}^{*}\left(k_{n}, n+1\right) \prod_{j_{n}=1}^{n+1} \mathcal{G}_{1}^{*}\left(t_{n}, j_{n}\right) \sum_{\mathrm{P}:(1, \ldots, n+1)=\cup_{i} X_{i}} \prod_{X_{i} \subset \mathrm{P}} g_{\left|X_{i}\right|}\left(X_{i}\right) \prod_{i=1}^{n+1} g_{1}^{0}(i) .
\end{aligned}
$$

In series expansion (32) the operator $\mathcal{N}_{\text {int }}^{*}\left(j_{1}, j_{2}\right)$ is defined according to formula (2) and the group of operators $\mathcal{G}_{1}^{*}(t)$ is defined by (1). For bounded interaction potentials series (32) is norm convergent on the space $\mathfrak{L}^{1}(\mathcal{H})$ under the condition that: $t<t_{0} \equiv\left(2\|\Phi\|_{\mathfrak{L}\left(\mathcal{H}_{2}\right)}\left\|g_{1}^{0}\right\|_{\mathfrak{L}^{1}(\mathcal{H})}\right)^{-1}$.

For marginal correlation functionals (28) the following statement is true [9]. Under conditions (21) and (31) on initial state (26) there exists a mean field limit of marginal correlation functionals (28) in the following sense:

$$
\lim _{\epsilon \rightarrow 0}\left\|\epsilon^{s} G_{s}\left(t, 1, \ldots, s \mid G_{1}(t)\right)-g_{s}\left(t, 1, \ldots, s \mid g_{1}(t)\right)\right\|_{\mathcal{L}^{1}\left(\mathcal{H}_{s}\right)}=0, \quad s \geq 2,
$$

where the limit marginal correlation functionals $g_{s}\left(t \mid g_{1}(t)\right), s \geq 2$, are represented by the expansions:

$$
g_{s}\left(t, 1, \ldots, s \mid g_{1}(t)\right)=\prod_{i_{1}=1}^{s} \mathcal{G}_{1}^{*}\left(t, i_{1}\right) g_{s}(1, \ldots, s) \prod_{i_{2}=1}^{s}\left(\mathcal{G}_{1}^{*}\right)^{-1}\left(t, i_{2}\right) \prod_{j=1}^{s} g_{1}(t, j), \quad s \geq 2,
$$

and, respectively, the limit one-particle density operator $g_{1}(t)$ is represented by series (32).

The proof of these statements is based on the validity of equality (20) for cumulants of asymptotically perturbed groups of operators (1) and the explicit structure of the generating operators of series expansions (28) of marginal correlation functionals and of series expansion (27).

We remark that limit marginal correlation functionals (32), (33) are a solution of the Cauchy problem of the quantum Vlasov hierarchy of nonlinear evolution equations [6], which describes a mean field asymptotic behavior of marginal correlation operators in case of arbitrary initial states, namely,

$$
\begin{aligned}
& \frac{\partial}{\partial t} g_{s}(t, 1, \ldots, s)=\sum_{i=1}^{s} \mathcal{N}^{*}(i) g_{s}(t, 1, \ldots, s)+ \\
& \operatorname{Tr}_{s+1} \sum_{i=1}^{s} \mathcal{N}_{\text {int }}^{*}(i, s+1)\left(g_{s+1}(t, 1, \ldots, s+1)+\sum_{\substack{\mathrm{P}:(1, \ldots, s+1)=X_{1} \cup X_{2}, i \in X_{1} ; s+1 \in X_{2}}} g_{\left|X_{1}\right|}\left(t, X_{1}\right) g_{\left|X_{2}\right|}\left(t, X_{2}\right)\right), \\
& \left.g_{s}(t)\right|_{t=0}=g_{s}^{0}, \quad s \geq 1,
\end{aligned}
$$

where we used notations similar to accepted above.

It should be noted that limit marginal correlation functionals (33) describe the process of the evolution of correlations of large quantum particle systems by means of a one-particle density operator in a mean field approximation. 
Similar to the derivation of kinetic equation (30) we establish that the one-particle density operator represented by series expansion (32) is a solution of the Cauchy problem of the Vlasovtype quantum kinetic equation with initial correlations:

$$
\begin{aligned}
& \frac{\partial}{\partial t} g_{1}(t, 1)=\mathcal{N}^{*}(1) g_{1}(t, 1)+ \\
& \operatorname{Tr}_{2} \mathcal{N}_{\text {int }}^{*}(1,2) \prod_{i_{1}=1}^{2} \mathcal{G}_{1}^{*}\left(t, i_{1}\right)\left(g_{2}(1,2)+I\right) \prod_{i_{2}=1}^{2}\left(\mathcal{G}_{1}^{*}\right)^{-1}\left(t, i_{2}\right) g_{1}(t, 1) g_{1}(t, 2), \\
& \left.g_{1}(t)\right|_{t=0}=g_{1}^{0}
\end{aligned}
$$

and consequently, for pure states we derive the Hartree-type equation with initial correlations. We point out that equation (34) is the non-Markovian quantum kinetic equation.

Thus, we established that a mean field behavior of processes of the creation of correlations and the propagation of initial correlations in condensed states are governed by kinetic equation (34).

Moreover, in the case under consideration the processes of the creation of correlations generated by dynamics of many-particle systems and the propagation of initial correlations are described by the constructed marginal functionals of the state (28) governed by the non-Markovian generalized kinetic equation with initial correlations (26).

\section{Conclusion}

In this paper it was been described the process of a creation and a propagation of correlations in quantum many-particle systems within the framework of the Cauchy problem of the quantum BBGKY hierarchy of nonlinear equations (11), (12). A nonperturbative solution for a sequence of marginal correlation operators is represented in the form of series 13 the generating operator of every term of which are corresponding-order cumulant (14) of groups of nonlinear operators (3). In case of initial state specified by a sequence of the marginal correlation operators that satisfy chaos property (15) the correlations generated by dynamics of large quantum particle system (16) is completely determined by the corresponding-order cumulants (4) of groups of operators (1). The obtained results can be extended to large quantum systems of bosons and fermions like in paper [6].

In case of initial states satisfied condition (15) a mean eld asymptotic behavior of the processes of a creation and a propagation of correlations was described. It was directly proved the property called the propagation of initial chaos (22), which underlies in mathematical derivation of effective evolution equations of systems of infinitely many particles [18].

It was been also considered the problem of the rigorous description of collective behavior of quantum many-particle systems by means of a one-particle (marginal) correlation operator that is a solution of the generalized quantum kinetic equation [23] with initial correlations [22], for instance, the initial correlations, characterizing the condensed states [1] or initial correlations that influence on ultrafast relaxation processes in plasmas [4].

In particular, such an approach to the derivation of the Vlasov-type quantum kinetic equation with initial correlations (34) from underlying dynamics governed by the generalized quantum kinetic equation with initial correlations (30) enables to construct the higher-order corrections to the mean field evolution of large quantum systems of particle. 
We note that in paper [24] other approach to the description of the propagation of initial correlations of many-particle systems in a mean field limit was developed, namely the process of the propagation of initial correlations was described within the framework of the evolution of marginal observables governed by the dual BBGKY hierarchy [21], 25].

\section{References}

[1] M.M. Bogolyubov, Lectures on Quantum Statistics. Problems of Statistical Mechanics of Quantum Systems, Rad. Shkola, Kyiv, 1949 (in Ukrainian).

[2] V.I. Gerasimenko, Hierarchies of quantum evolution equations and dynamics of many-particle correlations. In: Statistical Mechanics and Random Walks: Principles, Processes and Applications. N.Y.: Nova Science Publ., Inc., 233, 2012.

[3] I. Prigogine, Non-Equilibrium Statistical Mechanics, Wiley, New York, 1962.

[4] M. Bonitz, C. Henning, D. Block, Complex plasmas: a laboratory for strong correlations. Rep. Prog. Phys., 73, (2010), 066501 (29pp).

[5] V.I. Gerasimenko, V.O. Shtyk, Evolution of correlations of quantum many-particle systems. J. Stat. Mech. Theory Exp., 3, P03007, 24p., 2008.

[6] V.I. Gerasimenko, D.O. Polishchuk, Dynamics of correlations of Bose and Fermi particles. Math. Meth. Appl. Sci., 34, (1), 76, 2011.

[7] V.I. Gerasimenko, Evolution of correlation operators of large quantum particle systems. Methods Funct. Anal. Topology, 23, (2), 123, 2017.

[8] V.I. Gerasimenko, Processes of creation and propagation of correlations in quantum many-particle systems. Reports of NAS of Ukraine, 5, 58, 2016.

[9] V.I. Gerasimenko, On the description of quantum correlations by means of a one-particle density operator. Proc. IM NASU, 14, (1), 116, 2017.

[10] L. Saint-Raymond, Kinetic models for superfluids: a review of mathematical results. C.R. Physique, $\mathbf{5}, 65,2004$.

[11] D. Benedetto, F. Castella, R. Esposito and M. Pulvirenti, A short review on the derivation of the nonlinear quantum Boltzmann equations. Commun. Math. Sci., 5, 55, 2007.

[12] F. Pezzotti, M. Pulvirenti, Mean-field limit and semiclassical expansion of quantum particle system. Ann. Henri Poincaré. 10, 145, 2009.

[13] L. Erdös, B. Schlein, H.-T. Yau, Derivation of the cubic nonlinear Schrödinger equation from quantum dynamics of many-body systems. Invent. Math., 167, (3), 515, 2007.

[14] L. Erdös, B. Schlein, H.-T. Yau, Derivation of the Gross-Pitaevskii equation for the dynamics of Bose-Einstein condensate. Ann. of Math., 172, 291, 2010.

[15] N. Benedikter, M. Porta and B. Schlein, Effective Evolution Equations from Quantum Dynamics, SpringerBriefs in Mathematical Physics, 2016. 
[16] C. Boccato, S. Cenatiempo, B. Schlein, Quantum many-body fluctuations around nonlinear Schrödinger dynamics. Ann. Henri Poincaré, 18, (1), 113, 2017.

[17] M. Porta, S. Rademacher, C. Saffirio, B. Schlein, Mean field evolution of fermions with Coulomb interaction. J. Stat. Phys., 166, (6), 1345, 2017.

[18] F. Golse, On the dynamics of large particle systems in the mean field limit, In: Macroscopic and large scale phenomena: coarse graining, mean field limits and ergodicity, Lect. Notes Appl. Math. Mech., Springer, 3, 1, 2016.

[19] F. Golse, C. Mouhot, T. Paul, On the mean-field and classical limits of quantum mechanics. Commun. Math. Phys., 343, 165, 2016.

[20] H. Spohn, Kinetic equations from Hamiltonian dynamics. Rev. Modern Phys., 53, 600, 1980.

[21] V.I. Gerasimenko, Approaches to derivation of quantum kinetic equations. Ukrainian J. Phys. 54, (8-9), 834, 2009.

[22] V.I. Gerasimenko, Zh.A. Tsvir, On quantum kinetic equations of many-particle systems in condensed states. Physica A: Stat. Mech. Appl., 391, (24), 6362, 2012.

[23] V.I. Gerasimenko, Zh.A. Tsvir, A description of the evolution of quantum states by means of the kinetic equation. J. Phys. A: Math. Theor. 43, (48), 485203, 2010.

[24] V.I. Gerasimenko, New approach to derivation of quantum kinetic equations with initial correlations, Carpathian Math. Publ., 7, 38, 2015.

[25] V.I. Gerasimenko, Heisenberg picture of quantum kinetic evolution in mean-field limit. Kinet. Relat. Models, 4, (1), 385, 2011. 